\title{
Construction and characterization of a PDCD5 recombinant lentivirus vector and its expression in tumor cells
}

\author{
SHIYONG WANG ${ }^{1}$, XUE ZENG $^{1}$, YANPING LIU ${ }^{2}$, CHUNYAN LIANG $^{1}$, \\ HUI ZHANG ${ }^{1}$, CHANG LIU ${ }^{1}$, WEILI DU ${ }^{1}$ and ZHE ZHANG $^{1}$ \\ ${ }^{1}$ Department of Biotherapy and Laboratory of Biotherapy, The Fourth Affiliated Hospital of China Medical University, \\ Shenyang 110032; ${ }^{2}$ Department of Respiratory Medicine, Shenyang Weikang Hospital, Shenyang 110021, P.R. China
}

Received January 12, 2012; Accepted March 2, 2012

DOI: $10.3892 /$ or.2012.1756

\begin{abstract}
The function and mechanism of the programmed cell death 5 (PDCD5) gene is not completely understood, so it is necessary to build a stable and efficient PDCD5 recombinant lentiviral expression vector. The coding region of the PDCD5 gene was PCR amplified from pCMV-SPORT6. Next, the PDCD5 fragment and the pGC-FU vector were digested with the restriction enzyme AgeI and ligated by in-Fusion technology to build the pGC-FU-PDCD5 plasmid. Competent E. coli DH5 $\alpha$ cells were transformed and positive clones were identified by PCR. The PCR products were digested and sequenced. After sequencing, positive clones were selected to grow and propagate. The pGC-FU-PDCD5 plasmid DNA was purified and mixed with the pHelper1.0 and pHelper2.0 packaging plasmids. They were co-transfected into $293 \mathrm{~T}$ cells. The viral titer was measured by real-time PCR. The expression of GFP and PDCD5 was detected by both Western blotting and fluorescence microscopy. Additionally, the A498, ACHN, HCT116 and LoVo tumor cell lines were transfected with the virus supernatant, and PDCD5 expression was detected in these cells. The constructed pGC-FU-PDCD5 plasmid contained the correct PDCD5 gene sequence. Strong green fluorescence in both the cytoplasm and cell membrane was observed following transfection with purified pGC-FU-PDCD5 into 293 T cells. The transfection rate was greater than 95\%, and the expression of the PDCD5-GFP fusion protein was confirmed by Western blotting. The titer of the recombinant PDCD5 lentiviral condensed supernatant was measured to be $2 \times 10^{9} \mathrm{Tu} / \mathrm{ml}$. The transfection efficiencies of A498, ACHN and HCT116 cells were greater than $90 \%$. However, transfection efficiency of LoVo cells was lower but still greater than $70 \%$. The PDCD5-GFP fusion protein was stable in these transfected tumor cells, as detected by Western blotting. In conclusion, a PDCD5 recombinant lentiviral vector
\end{abstract}

Correspondence to: Dr Shiyong Wang, Department of Biotherapy and Laboratory of Biotherapy, The Fourth Affiliated Hospital of China Medical University, Shenyang 110032, P.R. China E-mail: sywang66@yahoo.com

Key words: programmed cell death 5 protein, human, genetic vectors, lentivirus, recombination, genetic, cell line, tumor was successfully constructed, and high expression of the plasmid was observed in tumor cells.

\section{Introduction}

PDCD5 is an apoptosis-related gene (1), which is widely expressed in normal cells. PDCD5 expression is lower in embryonic tissues than in adult tissues. While it is decreased in precancerous lesions, it is either dramatically reduced or sometimes even completely lost in a number of advanced cancers, including ovarian, chondrosarcoma, prostate, glioma, leukemia, and stomach. In fact, PDCD5 expression decreases with increased tumor stage, reduced differentiation status, and metastasis to lymph nodes (2-7). Therefore, decreased PDCD5 gene expression is associated with poor prognosis of ovarian carcinoma, chondrosarcoma, and glioma $(2,3,5)$. A combination of both in vitro and in vivo experiments confirmed that tumor cells expressing PDCD5 or treated with recombinant PDCD5 protein were more sensitive to chemotherapeutic drugs and UV irradiation. For example, mice with tumors expressing PDCD5 and treated with chemotherapy or radiation showed increased apoptosis, decreased tumor growth, and prolonged survival (8-12). These results support a role for PDCD5 as a tumor suppressor that influences treatment and prognosis. Moreover, a SNP rs1862214, located $35 \mathrm{~kb}$ upstream of the PDCD5 gene, was found to significantly correlate with susceptibility to lung carcinogenesis in a study of two groups, one German and one Italian. Compared with the frequent CC genotype, the variant GG or GC genotype carrier has a significantly increased risk of lung tumorigenesis. This risk was further increased in the advanced, lymph node metastasis, or short-term survival patients. PDCD5 mRNA was 2.4-fold lower in lung cancer tissue compared to normal lung (13). An analogous study found similar results for this SNP and its influence on susceptibility to chronic myeloid leukemia (14). Despite these findings, a correlation between this particular SNP and lung cancer susceptibility was not seen in a Japanese population (15).

On its own, PDCD5 does not induce apoptosis. Instead, it seems to be a pro-apoptotic factor that cooperates with chemotherapy or UV irradiation to cause cell death (8-12). In several studies, increased PDCD5 expression is accompanied by its translocation from the cytoplasm to the nucleus, a process that occurs prior to cell death. Thus, this nuclear translocation of 
PDCD5 may be an early apoptotic event playing an important role in regulating this process (16). Using both antisense gene knockdown and protein inhibition, PDCD5 was found to be a positive regulator of the apoptotic protein caspase-3. Additionally, rhPDCD5 had an effect on mitochondrial membrane potential, resulting in increased cytochrome $\mathrm{C}$ release. Thus, there seems to be amplification of the apoptotic response via a positive feedback mechanism (17). The PDCD5 protein is comprised of a core area and two free zones, the $\mathrm{N}$-terminus and the $\mathrm{C}$-terminus. The C-terminus of the PDCD5 protein is important for its uptake into the cell. PDCD5 can also carry other large proteins as cargo and enter the cell by endocytosis (18). Despite these studies, it is still unclear how PDCD5 actually works. It may be a substrate of the protein kinase CK2 (19) or it may play a dual role in the Tat-interactive protein (Tip60) pathway, in which PDCD5 binds to Tip60 and enhances its stability. The binding of PDCD5 to Tip60 is significantly increased after UV irradiation. PDCD5 increases Tip60-dependent K120 acetylation of p53 and participates in p53-dependent expression of apoptosis-related genes, such as Bax. This demonstrates the biological significance of the PDCD5-Tip60 interaction. That is, they cooperate with other molecules to accelerate DNA damage-induced apoptosis (20). There are many transcription factor binding sites found within the 1.1-kb region upstream of the PDCD5 transcriptional start site, indicating the PDCD5 plays an important role in gene transcriptional regulation (21). PDCD5 may also play a role in the protein translation process, but the mechanism is poorly understood. To date, most experiments have focused on transient expression of PDCD5 for functional studies. However, for gene therapy applications, sustained expression is required. In this study, we successfully constructed a PDCD5 lentiviral gene transfer system using a new carrier-lentiviral vector. We examined its transfection efficiency in four tumor cell lines. This study lays the foundation for in vitro and in vivo study on the biological function of PDCD5.

\section{Materials and methods}

Main reagents and instruments. Lentiviral vector pGC-FU (Fig. 1, the vector with the CMV promoter and enhanced green fluorescent protein gene, eGFP) and two packaging components, pHelper1.0 and pHelper2.0, were purchased from Shanghai Genechem Co. The pCMV-SPORT6 plasmid containing the human gene PDCD5 coding sequence was purchased from Open Biosystems Co. The E. coli DH5 $\alpha$ was a frozen strain obtained from the Human Genome Laboratory, China Medical University. The In-Fusion kit was purchased from BD Co. Plasmid extraction kit and DNA recovery kit were purchased from Qiagen Co., China. PCR kit and Taq DNA polymerase were purchased from Takara Co. The restriction endonuclease AgeI was from NEB Co. Agarose gel was purchased from Parkson Corp. DNA ladders (1 kb and $250 \mathrm{bp}$ ) were from Fermentas Co. ECL-Plus kit was purchased from Amersham Corp. Rabbit anti-PDCD5 (12456-1-AP, $14 \mathrm{kDa}$ ) was from the US PTG. Antibodies goat anti-rabbit (sc-2004), mouse anti-GFP (sc-9996), goat antimouse (sc-2005), and mouse anti-GAPDH (sc-32233, 1:5,000) were from Santa-Cruz. BCA protein assay kit (cat. no. 23225) was a HyClone-Pierce product. Prestained protein marker was from Zhongjing Co. Plus-20 centrifugal ultrafiltration device was from Millipore Co. Quantitative PCR instrument IQ5 and electrophoresis instrument were from Bio-Rad. SDS-PAGE electrophoresis instrument was from the Shanghai Qiagen Co.

Cell lines and cell culture. Human cancer cell lines HCT116, LoVo, ACHN, A498, HeLa, H1299, and A375 were purchased from the Shanghai Cell Bank of Chinese Academy of Sciences. The HCT116 cells were cultured with McCoy's 5A medium, the SW620 cells with Leibovitz's L-15 medium, the A498 and A375 cells were cultured with DMEM medium, and the others were cultured with RPMI-1640 medium at $37^{\circ} \mathrm{C}$ and $5 \% \mathrm{CO}_{2}$. All media used for cell culture were supplemented with $10 \%$ fetal calf serum. Cells in their logarithmic growth phase were used for experiments. Media and fetal calf serum were purchased from Gibco.

Primer design and synthesis. Referring to GenBank gene sequences, the software Primer Premier 5.0 was used to design primers, which were synthesized by Takara. The GenBank PDCD5 sequence number is no. NM_004708. The upstream primer was: PDCD5-AgeI-F: GAGGATCCCCGGGTACCGGT CGCCACCATGGCGGACGAGGAGCTTG, which included the exchange pairing bases, the AgeI restriction enzyme cutting site (underlined), the expressed enhancing sequence (double underlined), and the target gene $5^{\prime}$ sequence so as to amplify the target gene. The downstream primer was: PDCD5-AgeI-R: TCACCATGGTGGCGACCGGATAATCGTCATCTTCATC AGAGTC, which included the exchange pairing bases, the AgeI restriction site (underlined, the $\mathrm{T}$ base was removed in order to adjust the reading frame), and the target gene $3^{\prime}$ end partial sequence for PCR amplification. Positive clones were identified by PCR, and the PCR product was sequenced by the primers containing the PDCD5-Age I-F upstream primer and downstream primer CGTCGCCGTCCAGCTCGACCAG, which is located at the N-terminus of the eGFP gene (see the pGC-FU plasmid structure above). The GFP primers for virus titer detection are as follows: upstream primer: 5'-TGCTTCAG CCGCTACCC-3', downstream primer: 5'-AGTTCACCTTGAT GCCGTTC-3', and the predicted amplified fragment size is 286 bp. $\beta$-actin was used as an internal reference, and its upstream primer: 5'-GGACATCCGCAAAGAC-3', downstream primer: 5'-AAAGGGTGTAACGCAACTA-3', and the amplified fragment size is $302 \mathrm{bp}$.

PCR for the PDCD5 gene fragment amplification. The PDCD5 coding region was amplified using the pCMV-SPORT6 plasmid as a template and PDCD5-Age I-F and PDCD5-Age I-R primers. The reaction mixture volume of $20 \mu \mathrm{l}$, contained $0.4 \mu \mathrm{l}$ upstream and downstream primers, respectively, $\mathrm{ddH}_{2} \mathrm{O} 14.7 \mu \mathrm{l}$, 10X buffer $2 \mu \mathrm{l}, 1.5 \mathrm{mM} \mathrm{MgCl}_{2} 0.5 \mu \mathrm{l}, 2.5 \mathrm{mM}$ dNTPs $0.8 \mu \mathrm{l}$, pfu polymerase $0.2 \mu \mathrm{l}$, and $10 \mathrm{ng} / \mu \mathrm{l}$ template $1 \mu \mathrm{l}$. The $\mathrm{PCR}$ reaction conditions are as follows: $94^{\circ} \mathrm{C}$ pre-denaturing for $30 \mathrm{sec}, 94^{\circ} \mathrm{C}$ denaturing for $30 \mathrm{sec}, 55^{\circ} \mathrm{C}$ annealing for $30 \mathrm{sec}, 72^{\circ} \mathrm{C}$ extending for $1 \mathrm{~min}$, and $72^{\circ} \mathrm{C}$ for a final $10 \mathrm{~min}$. The total PCR process was 30 cycles. The PCR products were separated by agarose gel electrophoresis, and the PDCD5 coding region fragment DNA was extracted and purified using DNA gel extraction kit and DNA recovery kit separately.

Construction of the $p G C-F U-P D C D 5$ recombinant plasmid and sequencing. The purified PDCD5 fragment and pGC-FU 


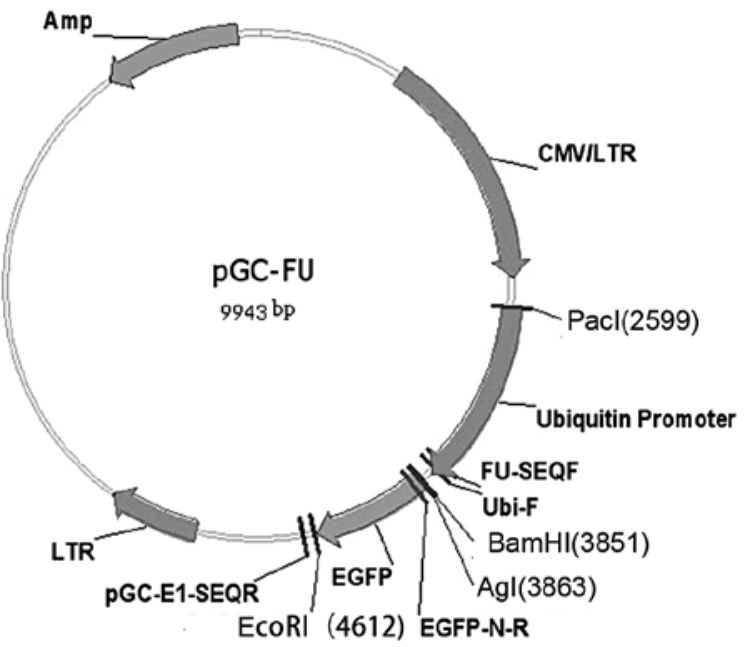

Figure 1. The pGC-FU structure of eukaryotic expression vector (provided by Shanghai Genechem Co.).

gene plasmid (see the description above) were digested separately with AgeI. The reaction volume was $50 \mu$ l containing the purified plasmid $2 \mu \mathrm{l}$, or $1 \mu \mathrm{g} / \mu \mathrm{l}$ DNA $1 \mu \mathrm{l}$, 10X buffer $5 \mu \mathrm{l}$, 100X BSA $0.5 \mu \mathrm{l}, 10 \mathrm{U} / \mu \mathrm{l}$ AgeI enzyme $1 \mu \mathrm{l}, \mathrm{ddH}_{2} \mathrm{O} 41.5 \mu \mathrm{l}$, mixed at $37^{\circ} \mathrm{C}$ for $1 \mathrm{~h}$.

Using the In-Fusion kit, the PDCD5 fragment was ligated into the pGC-FU expression vector which was digested in advance with AgeI, the total ligation reaction volume was $20 \mu$ l containing $100 \mathrm{ng} / \mu 1$ vector DNA $2 \mu \mathrm{l}, 100 \mathrm{ng} / \mu \mathrm{l}$ PDCD5 fragment $2 \mu \mathrm{l}$, 10X In-Fusion exchange enzyme buffer $1.0 \mu \mathrm{l}$, exchange enzyme In-fusion $0.5 \mu \mathrm{l}, \mathrm{ddH}_{2} \mathrm{O} 13.5 \mu \mathrm{l}$, two control groups were used, the digested vector or PDCD5 fragment DNA was added, the reaction was at $23^{\circ} \mathrm{C}$ for $15 \mathrm{~min}$, then was warmed at $42^{\circ} \mathrm{C}$ for $15 \mathrm{~min}$, and the DNA cloning ligation system was prepared.

The DH5 $\alpha$ competent bacteria suspension was added into sterile Eppendorf microcentrifuge tubes, $200 \mu \mathrm{l}$ in each tube, clone ligation fluid $10 \mu \mathrm{l}$ per tube was added, the tubes were mixed gently, and placed on ice for $30 \mathrm{~min}$, then at $42^{\circ} \mathrm{C}$ in a circulating water bath for $90 \mathrm{sec}$. The tubes were quickly transferred to an ice bath 1-2 min for cooling, and $800 \mu$ l LB medium was added each tube, the tubes were heated in $37^{\circ} \mathrm{C}$ water bath, and transferred to a $37^{\circ} \mathrm{C}$ shaker. The bacteria in the tubes were incubated for $45 \mathrm{~min}$ and then $150 \mu \mathrm{l}$ of the transformed competent cells were transferred to LB agar plates which contained $20 \mathrm{mM} / 1 \mathrm{MgSO}_{4}$ and $100 \mu \mathrm{g} / \mathrm{ml}$ AMP. These plates were placed at room temperature until the liquid was absorbed, the plates were inverted and cultured at $37^{\circ} \mathrm{C}$ for $16 \mathrm{~h}$, and the positive clones were identified with PCR method.

The E. coli clones were cultured in LB medium, and the plasmid was extracted by mini-prep plasmid extraction kit, the target gene PDCD5 was amplified by PCR, then the correct recombinant plasmid was identified. The $\mathrm{PCR}$ reaction conditions were as follows: $94^{\circ} \mathrm{C}$ predenaturing for $30 \mathrm{sec}, 94^{\circ} \mathrm{C}$ denaturing for $30 \mathrm{sec}, 60^{\circ} \mathrm{C}$ annealing for $30 \mathrm{sec}, 72^{\circ} \mathrm{C}$ extending for $30 \mathrm{sec}$, $72^{\circ} \mathrm{C}$ for $6 \mathrm{~min}$, and the total PCR reaction was 30 cycles. The PDCD5-Age I-F and EGFP-N-R primers were used to set up the PCR reaction, total volume was $20 \mu \mathrm{l}$, and the reaction mixture is shown in the methods above. The positive bacteria colonies were inoculated into $\mathrm{LB}$ medium at $37^{\circ} \mathrm{C}$ for $16 \mathrm{~h}$, and then, after adding glycerol, were stored at $-80^{\circ} \mathrm{C}$. Aliquots $(200 \mu \mathrm{l})$ of each were sent for DNA sequencing by Shanghai Genechem Co.

Transfection of the PDCD5 recombinant lentiviral plasmid in $293 T$ cells. Twenty-four hours before transfection, 293T cells growing in the logarithmic phase were chosen and propagated. Cell number was adjusted to $1.2 \times 10^{6} / \mathrm{ml}$ with $10 \%$ FCS DMEM medium. Cells $(20 \mathrm{ml})$ were placed into $15 \mathrm{~cm}$ diameter dish and cultured at $37^{\circ} \mathrm{C}$ with $5 \% \mathrm{CO}_{2}$. The cells were transfected at $70 \%-80 \%$ confluence, and $2 \mathrm{~h}$ before transfection, the medium was changed to serum-free medium.

The recombinant pGC-FU-PDCD5 vector and two packaging components pHelper1.0 and pHelper2.0 DNAs were extracted from the positively transformed bacteria. This endotoxin-free plasmid DNA was purified and dissolved in TE buffer. The plasmid DNA concentration was measured with UV absorption A260/A280 values (normally between 1.8-2.0). Recombinant pGC-FU-PDCD5 vector $(20 \mu \mathrm{g})$ and $15 \mu \mathrm{g}$ pHelper1.0 and $10 \mu \mathrm{g}$ pHelper2.0 were added into a sterile centrifuge tube and then mixed with corresponding volume of Opti-MEM. The total volume was adjusted to $2.5 \mathrm{ml}$, and the tubes were incubated at room temperature for $5 \mathrm{~min}$. The $293 \mathrm{~T}$ cells were then co-transfected with the DNAs via Lipofectamine 2000 according to the manufacturer's instructions (Invitrogen cat. no. 11668-019). After 8-h transfection, the cells were washed gently with PBS, the medium was replaced with complete culture medium, and the cells were continuously cultured for $48 \mathrm{~h}$. The cells supernatant was collected and condensed with the Centricon Plus-20 Product kit (www.millipore.com) to obtain a high titer lentivirus concentration. 293T cells were used to measure the supernatant virus titer. The transfected 293T cells were collected, and the PDCD5 expression was analyzed by Western blotting.

Western blot analysis on the PDCD5 expression. The transfected and untransfected 293T or tumor cells were harvested and a specific volume of ice cold $2 \mathrm{X}$ lysis buffers $(100 \mathrm{mM}$ Tris-HCI, 2\% mercaptoethanol, 20\% glycerol, 4\% SDS) was added. Cells were incubated on ice for 10-15 min, sonicated ( $200 \mathrm{~W}$ x 4 times, every $5 \mathrm{sec}$, interval $2 \mathrm{sec}$ ) briefly, centrifuged at $4^{\circ} \mathrm{C}$ at $12,000 \mathrm{~g} \mathrm{x} 15 \mathrm{~min}$. The supernatant was collected, the protein concentration was adjusted to $2 \mu \mathrm{g} / \mu \mathrm{l}$, and the supernatant was stored at $-80^{\circ} \mathrm{C}$ for later use. After denaturing, the proteins were separated using conventional gel electrophoresis of $12 \%$ SDS-PAGE, and transferred to PVDF membrane with $400 \mathrm{~mA}$ constant current for $2 \mathrm{~h}$. The membrane was then blocked with 5\% milk TBST solution overnight, an appropriate dilution of primary antibody was added at room temperature for $2 \mathrm{~h}$, then the membrane was washed with TBST for $10 \mathrm{~min}$ $\mathrm{x} 3$ times, then a secondary antibody was added at 1:5,000 dilution at room temperature for 2 -h incubation, the membrane was washed with TBST for 10 min $x 3$ times, and detected with the ECL chemiluminescence kit in the dark for X-ray imaging.

Detection of the recombinant lentiviral titer by real-time PCR. The viral titer was measured by GFP expression; the $\beta$-actin gene was used as internal control. 293T cells $\left(5 \times 10^{4}\right)$ were placed into each well of a 24-well plate for 24-h culture, and the condensed virus stock solution $10 \mu \mathrm{l}$ (see the methods above) was added into an Eppendorf tube containing $90 \mu \mathrm{l}$ medium, then mixed and diluted 10 times. Seven concentrations were divided in total, and 


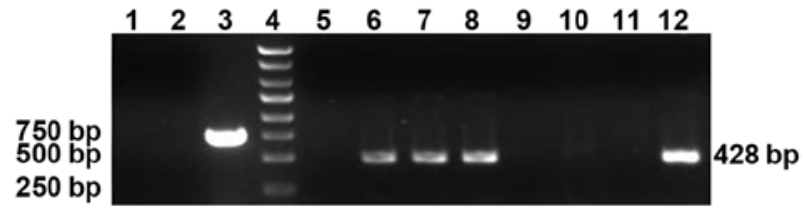

Figure 2. The pGC-FU-PDCD5 expression vector identified in bacterial clones by PCR. Lane 1, negative control $\left(\mathrm{ddH}_{2} \mathrm{O}\right)$; lane 2 , negative control (pGC-FU empty vector); lane 3, positive control (GAPDH); lane 4, marker: 5, 3, 2, 1.5 and $1 \mathrm{~kb}, 750,500$ and $250 \mathrm{bp}$; lanes 5-12, eight pGC-FU-PDCD5 transformed clones, of which, 6, 7, 8 and 12 were positive clones.

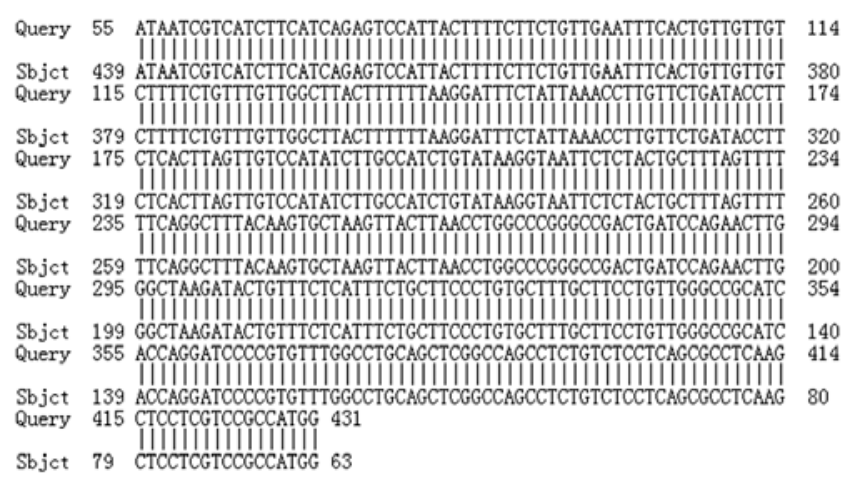

Figure 3. The PDCD5 gene sequencing in the pGC-FU-PDCD5 vector after positive clones were selected.

added separately into the wells in which the 293T cells were cultured. After 48-h culture, the complete culture medium was added. Four days later, the fluorescence expression was examined. The supernatant was discarded after washing with PBS. Total RNA in the cells was extracted with TRIzol according to the Invitrogen instructions. The reverse transcriptase (RT) reaction was then performed for cDNA synthesis (Promega M-MLV instruction). The RT reaction volume was $11 \mu \mathrm{l}$ containing $5 \mathrm{X}$ RT buffer $4 \mu \mathrm{l}, 10 \mathrm{mM}$ dNTPs $2 \mu \mathrm{l}$, RNasin 0.5 $\mu 1$, M-MLV-RTase $1 \mu 1$, DEPC $\mathrm{H}_{2} \mathrm{O} 3.5 \mu 1$, the reaction was first warmed at $42^{\circ} \mathrm{C}$ for $1 \mathrm{~h}$, then the RTase was inactivated at $70^{\circ} \mathrm{C}$ for $10 \mathrm{~min}$. The synthesized cDNA was used for realtime PCR. The real-time PCR was set up in the Bio-Rad IQ5: SYBR Premix Ex Taq $10 \mu 1,5 \mu \mathrm{M}$ upstream and downstream primers each $1.0 \mu \mathrm{l}$, cDNA $1.0 \mu \mathrm{l}, \mathrm{ddH}_{2} \mathrm{O} 7.5 \mu \mathrm{l}$, the reaction conditions were as follows: $95^{\circ} \mathrm{C}$ predenaturing $15 \mathrm{sec}, 95^{\circ} \mathrm{C}$ denaturing $5 \mathrm{sec}, 60^{\circ} \mathrm{C}$ annealing $30 \mathrm{sec}, 60^{\circ} \mathrm{C}$ extension for $30 \mathrm{sec}$, total reaction was 40 cycles. The melting curve was: $95^{\circ} \mathrm{C} 1 \mathrm{~min}, 55^{\circ} \mathrm{C} 1 \mathrm{~min}$. From 55 to $95^{\circ} \mathrm{C}, 0.5^{\circ} \mathrm{C}$ was increased at each step, and maintained for $30 \mathrm{sec}$, total was 81 cycles, while the $\mathrm{OD}$ values were read at each step.

Transfection of the PDCD5 recombinant lentivirus in tumor cells. When A498, ACHN, HCT116 and LoVo tumor cells were grown at logarithmic phase, $5 \times 10^{4} \mathrm{cells} / \mathrm{ml}$ were placed into each well of a 6-well plate, these wells were divided into the virus group, empty vector group, and the blank cell group. These cells were cultured for $12 \mathrm{~h}$ and infected with the virus at 30-50\% confluence. Based on the pre-established experimental multiplicity of infection (MOI) of 100,10,10 and 10, respectively, the same amount of virus, empty vector, and culture medium was added for each well; the supernatant was discarded after 12-h culture and replaced with the complete medium. After 4-day transfection, cells were counted under bright field microscopy, the green fluorescent protein positive cells were counted under the fluorescent vision field, and the transfection efficiency was calculated. Cells were collected for protein detection when transfection efficiency reached $>80 \%$.

\section{Results}

The $p G C-F U$-PDCD5 plasmid construction. The PCR reaction with pCMV-SPORT6 plasmid as a template was set up, and the expected PCR product size of $421 \mathrm{bp}$ for the PDCD5 coding sequence was obtained after PCR. This product sequence contained AgeI restriction site(s). The PDCD5 PCR product and the pGC-FU plasmid were then digested separately with AgeI, and the pGC-FU-PDCD5 vector was constructed with In-fusion enzyme ligation. The competent $E$. coli DH5 $\alpha$ was transformed, and four positive bacteria clones containing a fusion gene composed of PDCD5 coding sequence and eGFP gene N-terminal sequence fragment were identified using PCR. The product size was 482 bp (Fig. 2), the recombinant pGCFU-PDCD5 was confirmed by sequencing, and the PDCD5 sequence length was $377 \mathrm{bp}$. This sequence was consistent with the human PDCD5 gene sequence in GenBank (Fig. 3).

Transfection of the PDCD5 recombinant lentiviral plasmid in 2937 cells and expression of GFP and PDCD5. The pGCFU-PDCD5 recombinant plasmid and packaging plasmids pHelper1.0 and pHelper 2.0 were co-transfected in 293T cells. GFP fluorescence was detected via microscopy after 12-h transfection, and the expression reached a peak at $48 \mathrm{~h}$ posttransfection. The transfection rate was $>95 \%$ (Fig. 4). GFP and
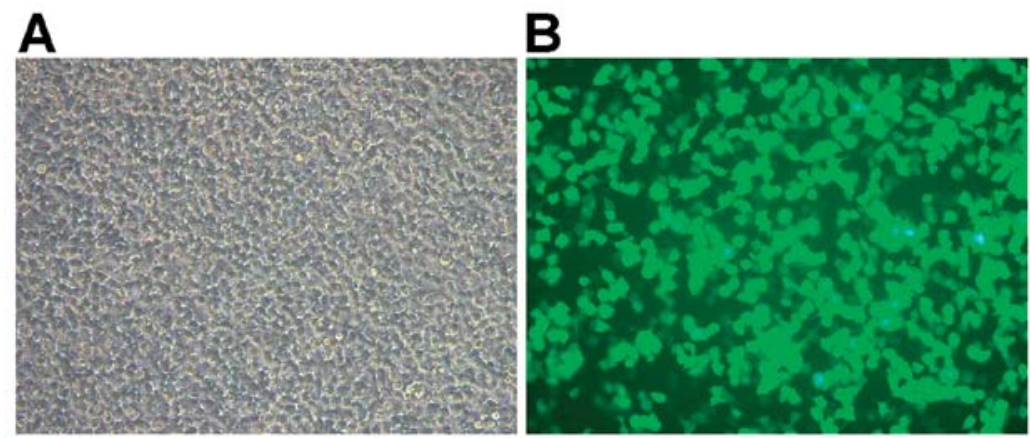

Figure 4. The GFP expression after 48-h transfection of the PDCD5 recombinant lentiviral plasmid in 293T cells (x200). (A) Bright field. (B) Dark field. 


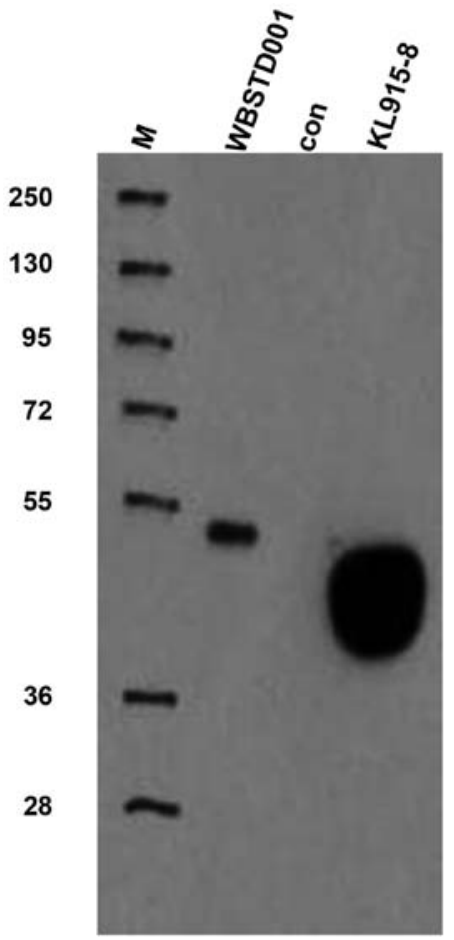

Figure 5. The GFP detection by Western blotting after the PDCD5 recombinant lentiviral plasmid transfected $48 \mathrm{~h}$ in $293 \mathrm{~T}$ cells. Marker: 28, 36, 55, 72, 95 , 130 and $250 \mathrm{kDa}$; WBSTD001, standard, fused GFP gene product, $48 \mathrm{kDa}$; con, 293T cells; KL915-8, positive clone transfected 293T; 1st Ab mouse antiGFP, dilution 1:2,000; 2nd goat anti-mouse, dilution 1:5,000, the assays were independent in duplicate.
PDCD5 were detected by Western blotting, and a specific band size between 36 and $55 \mathrm{kDa}$ was uniquely observed in PDCD5 recombinant lentiviral transfected $293 \mathrm{~T}$ cells. This was consistent with PDCD5-GFP fusion protein size, indicating that the PDCD5 gene fused with GFP and could be co-expressed in 293T cells. However, the corresponding band was not detected in other cells (Figs. 5 and 8). The PDCD5 band signal detected was very strong with a specific band (Fig. 8).

PDCD5 recombinant lentiviral packaging and determination of the virus titer. The pGC-FU-PDCD5 recombinant plasmid and packaging plasmids pHelper1.0 and pHelper2.0 were co-transfected in $293 \mathrm{~T}$ cells. The virus supernatant was then collected, condensed, and diluted 10-fold and divided into seven concentrations. The $293 \mathrm{~T}$ cells were placed into the culture plates and collected after 4 days. Total RNA was extracted, the cDNA was obtained by reverse transcription reaction, and the real-time PCR assay was conducted. The difference of $\mathrm{Ct}$ values was compared between the control and test group in order to determine the titer concentration. $\mathrm{Ct}$ values $>2.0$ were considered to be significantly different. In this study, there was a 2.0 difference in $\mathrm{Ct}$ value between the 1.00E-05 $\mu \mathrm{l}$ sample group and control group, indicating that the virus particles in the 1.00E-05 $\mu \mathrm{l}$ sample group are still expressed. Assuming that there was at least one virus particle in this group, each $\mu \mathrm{l}$ supernatant viral titer was at least $1 /(1.00 \mathrm{E}-05) \times 20 \mu \mathrm{l}$ $=2.00 \mathrm{E}+6 \mathrm{Tu} / \mu 1$, the virus titer was $2 \times 10^{9} \mathrm{Tu} / \mathrm{ml}$ (Fig. 6 and Table I). The melting curve represents the amplification reaction
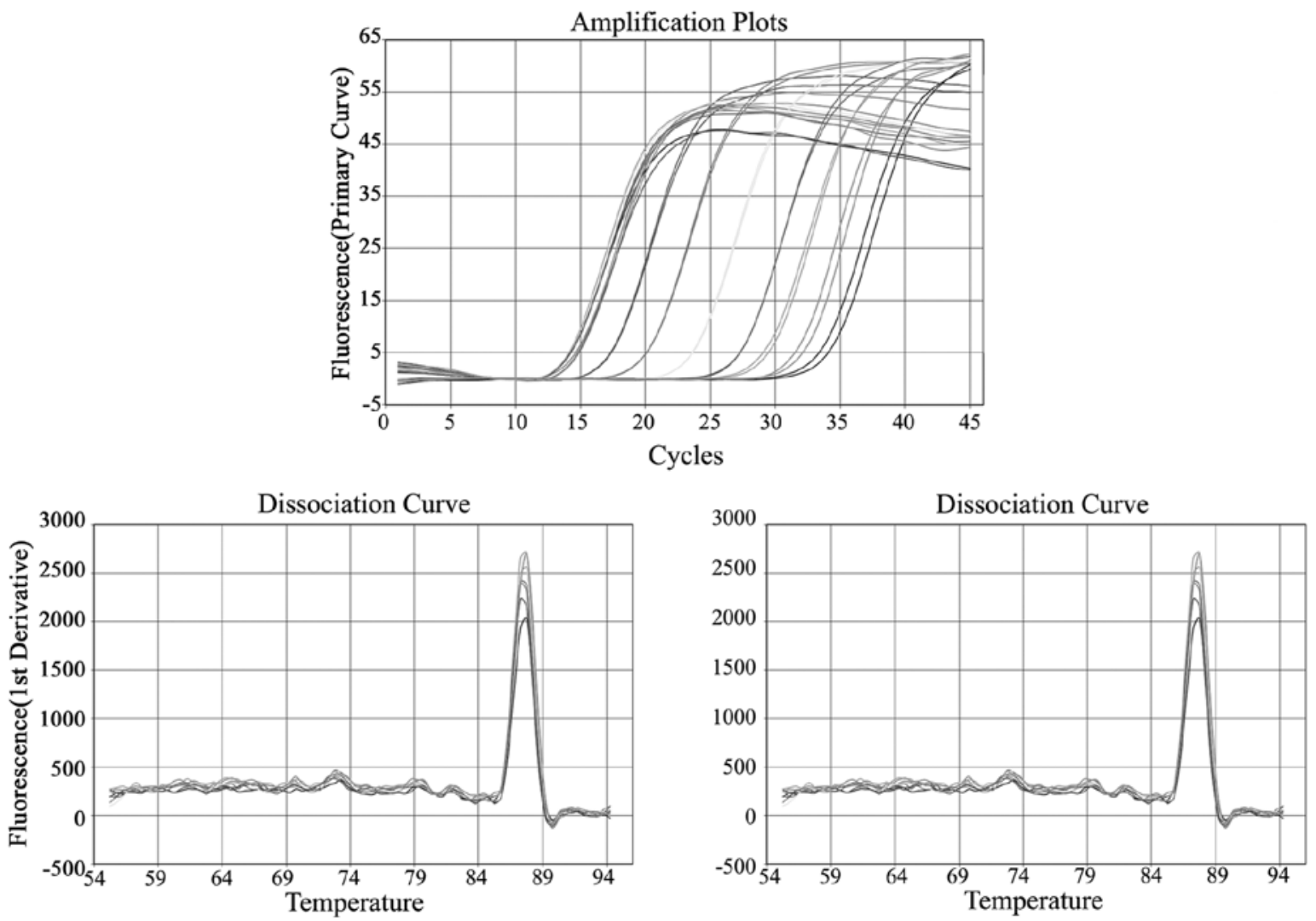

Figure 6. Measurement of the PDCD5 lentiviral amplification and melting curves by real-time PCR. The top is GFP gene and $\beta$-actin gene real-time PCR amplification curve, here GFP gene expression was used to replace the PDCD5 expression, $\beta$-actin gene was as an internal control, the different lines represent different virus dilutions. Lower panels, left and right, the melting curves represent the GFP and $\beta$-actin, respectively. 
Table I. Analysis of $\mathrm{Ct}$ values of different concentrations of virus infection and expression.

\begin{tabular}{lccrr}
\hline Sample group & $\mathrm{Ct}_{\text {actin }}$ mean & $\mathrm{Ct}_{\mathrm{GFP}}$ mean & $\Delta \mathrm{Ct}$ & $\Delta \Delta \mathrm{Ct}$ \\
\hline Con & 14.22 & 34.50 & 20.28 & 0.00 \\
$10 \mu \mathrm{l}$ & 14.43 & 15.01 & 0.58 & 19.70 \\
$1 \mu \mathrm{l}$ & 14.73 & 17.71 & 2.98 & 17.30 \\
$10^{-1} \mu \mathrm{l}$ & 14.27 & 20.75 & 6.48 & 13.80 \\
$10^{-2} \mu \mathrm{l}$ & 14.24 & 24.11 & 9.87 & 10.41 \\
$10^{-3} \mu \mathrm{l}$ & 14.22 & 27.81 & 13.59 & 6.69 \\
$10^{-4} \mu \mathrm{l}$ & 14.18 & 30.01 & 15.83 & 4.45 \\
$10^{-5} \mu \mathrm{l}$ & 14.82 & 32.53 & 17.71 & 2.57 \\
\hline
\end{tabular}

$\Delta \mathrm{Ct}, \mathrm{Ct}_{\mathrm{GFP}}$ mean - $\mathrm{Ct}_{\text {actin }}$ mean; $\Delta \Delta \mathrm{Ct}, \Delta \mathrm{Ct}_{\mathrm{con}}{ }^{-} \Delta \mathrm{Ct}$.

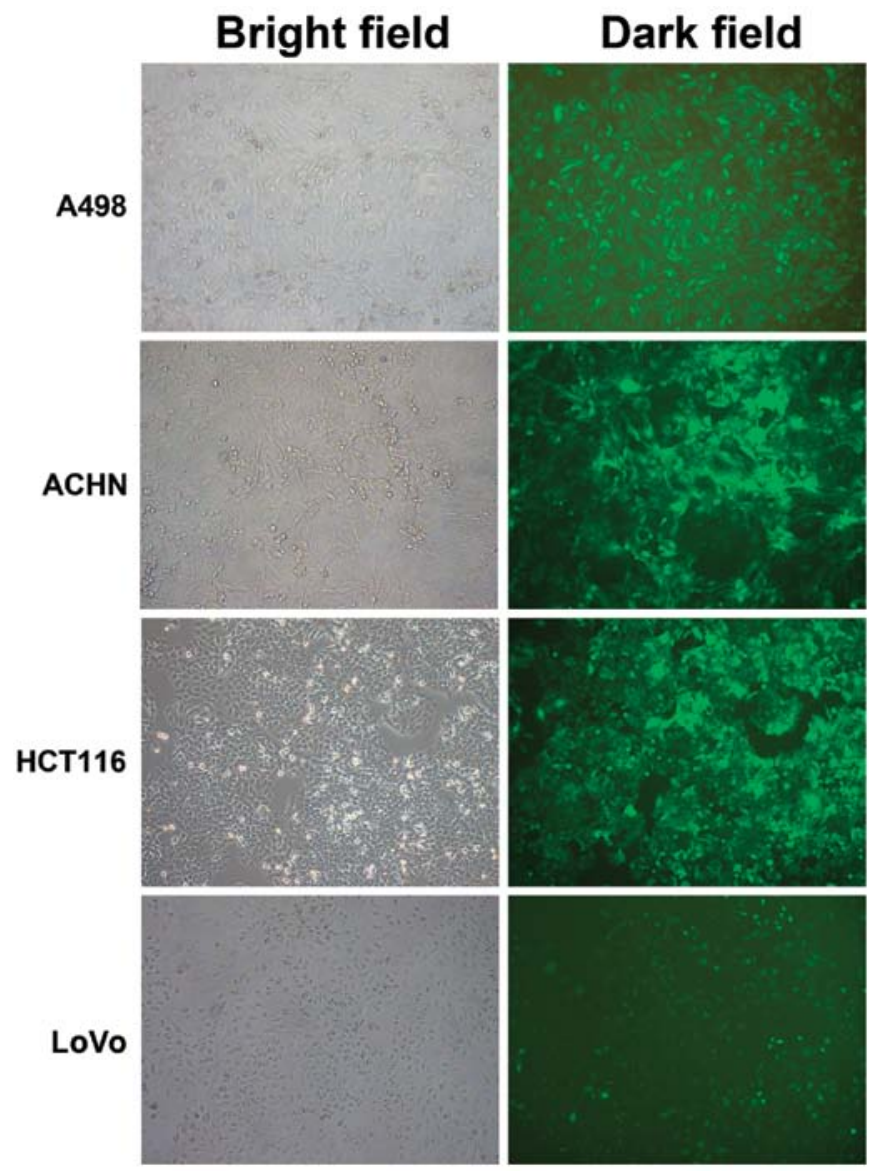

Figure 7. Expression of the PDCD5 recombinant lentivirus in 4 tumor cell lines after 72-h transfection (x100).

quality control. Impure peaks did not appear in the figure, nor did they appear in the broad abnormal peak, indicating that the experiment is not contaminated, and no primer dimer or nonspecific amplification was detected. The point for fluorescence intensity change is the peak value melting Tm.

Transfection of the recombinant lentiviral plasmid in four tumor cell lines and expression of GFP. The PDCD5 recom-

\begin{abstract}
1

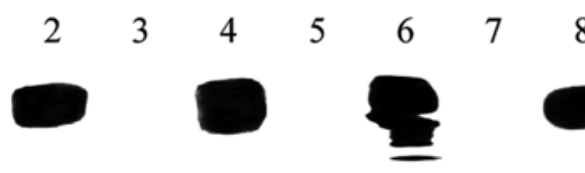

8

Figure 8. Transfection of the PDCD5 recombinant lentiviral plasmid in different tumor cell lines, the protein expression was detected by Western blotting (lanes 1 and 2, A498 cells pre- or post-transfection; lanes 3 and 4, ACHN cells pre- or post-transfection; lanes 5 and 6 , HCT116 cells pre- or post-transfection; lanes 7 and 8, LoVo cells pre- or post-transfection); 1st rabbit anti-PDCD5, dilution 1:200; 2nd goat anti-rabbit, dilution 1:5,000. The experiments were independent in duplicate.
\end{abstract}

binant lentivirus was successfully transfected and expressed constitutively in human renal carcinoma cells A498 and ACHN, and colon cancer cells HCT116 and LoVo. The expression of GFP was observed in the experimental group as well as the empty vector group under fluorescence microscope examination. Following recombinant lentivirus transfection for 3-7 days in A498, ACHN, HCT116 cells, the transfection rate was $>90 \%$. The transfection rate in the LoVo cells was lower, but $>70 \%$ (Fig. 7). The blank control group was negative.

Detection of the PDCD5 expression in tumor cell lines by Western blotting. After transfection with the recombinant lentivirus in A498, ACHN, HCT116, and LoVo cells, the cells were lysed, collected, and separated by $12 \%$ SDS-PAGE electrophoresis. Compared with the previous untransfected cells, a characteristic band 36-55 kDa was observed by Western blotting in LoVo, A498, HCT116 and ACHN cells, its size was consistent with the PDCD5-GFP fusion protein (Fig. 8), indicating that the PDCD5 gene in the recombinant PDCD5 lentiviral plasmid can be expressed after transfection in A498, ACHN, HCT116, and LoVo cells. The expressed specific band range was found to be wider especially in the HCT116 cells, which may be due to higher recombinant lentivirus infection efficiency, or may be due to the target gene expression in these cells being more potent than it is in other cells.

\section{Discussion}

PDCD5 is a pro-apoptotic gene (1). It will be easier to study the function of PDCD5 if the gene can be expressed in target cells via an effective vector. In addition, if expressed alongside certain apoptotic factors, the PDCD5 gene may be a good target gene for gene therapy. The adenovirus vector is currently widely used. However, it can only be transiently expressed, has immunogenicity, its carrier capacity is small, the titer of virus particles is lower, and it cannot meet clinical needs. As the only non-human disease associated virus, the adeno-associated virus is replication-defective. That is, its replication depends on the presence of helper virus, and the pathogenic capacity is weaker. It can undergo site-specific integration with a wide host range. The expression of the target gene after integration is long-term and stable, with better thermal stability, and acid-alkaline-proof. It also has a small packaging capacity and cannot be produced on a large scale. High-profile oncolytic virus results in natural infection, replication, and dissolved cell capacities. The virus can proliferate and dissolve tumor cells once infected. 
After cell lysis, the released virus particles can infect other cells and kill them. Hence, we need to improve the oncolytic virus targeting ability, avoiding the situation where it can be combined with natural receptors to attack normal cells (22).

Lentiviral vector derived from HIV and other 'suicide' viruses will no longer re-infect other cells after its first infection. It is also unable to produce new virus particles in host cells (23). As a new viral vector, the lentivirus has been widely developed in recent years. Its virulence gene was replaced by the exogenous target gene. The lentivirus is a pseudovirus since its envelope was the vesicular stomatitis virus VSV-G protein, which can avoid the infected recovery after homologous recombination. Lentiviral vector integrates into the host cell genome, and the foreign gene can be expressed stably and constitutively (24). It can also infect dividing and non-dividing cells, such as nerve cells, hematopoietic stem cells, and liver cells (25). The probability of lentiviral vector gene recombination is higher, the exogenous recombinant is stable, not easy to lose, and easy to be amplified in large amounts (26).

In this study, we first constructed the PDCD5 recombinant lentiviral expression vector. Using molecular cloning techniques such as PCR and DNA sequencing, the human PDCD5 lentiviral expression plasmid was successfully constructed, and high titer viral particles were obtained after plasmid transfection in packaging cells. The PDCD5-GFP expression rate reached more than $90 \%$ when human A498, ACHN, and HCT116 tumor cells were infected. The expression rate was lower in LoVo cells, but still more than $70 \%$. Furthermore, it was only highly expressed in the transfected tumor cell lines, and not expressed in non-transfected cells.

The constructed PDCD5 lentiviral recombinant vector will be used to study the function of the target gene after its stable transfection in tumor cell lines. This will allow for a detailed examination of the mechanism of PDCD5, including its relationship to apoptotic genes. It is also necessary to establish an animal model for human cancer gene therapy and clinical drugsensitizing treatment strategy. As a general vector, there are still some common problems with lentivirus. These include biological safety, transfection efficiency, stable expression problems, and low harvest. The third generation of the lentiviral vector has been developed. The second generation was self-inactivating due to deletion of the HIV-1 LTR regulatory element in the downstream region. Thus, the necessary packaging signal cannot be transcribed for the vector. The second generation had higher safety than the first one. The third generation retains the selfinactivating feature and also includes an inducible gene, such as tetracycline (tet), allowing for regulated expression in cells (27). If the PDCD5 gene was used to treat a tumor, it would be preferable to select a tumor-specific promoter or enhancer, such as hTERT, to insert into the upstream region of the gene. This would be important for virus amplification, so that gene transcription can be well controlled $(28,29)$. Finally, specific receptors might be able to be used to increase the targeting capacity of the viral envelope.

\section{Acknowledgements}

This study was supported by Natural Science Foundation of Liaoning Province (no. 201102296) and Scientific Research
Foundation for returned overseas scholar (2004-527) provided by Ministry of Education. We appreciate the valuable comments from other members of our laboratories. Shanghai Genechem Company provided some support and help.

\section{References}

1. Liu H, Wang Y, Zhang Y, et al: TFAR19, a novel apoptosis related gene cloned from human leukemia cell line TF21, could enhance apoptosis of some tumor cells induced by growth factor withdrawal. Biochem Biophy Res Commun 254: 203-210, 1999.

2. Zhang X, Wang X, Song X, et al: Clinical and prognostic significance of lost or decreased PDCD5 expression in human epithelial ovarian carcinomas. Oncol Rep 25: 353-358, 2011.

3. Chen $\mathrm{C}$, Zhou H, Xu L, et al: Prognostic significance of down-regulated expression of programmed cell death 5 in chondrosarcoma. J Surg Oncol 102: 838-843, 2010.

4. Du YJ, Xiong L, Lou Y, Tan WL and Zheng SB: Reduced expression of programmed cell death 5 protein in tissue of human prostate cancer. Chin Med Sci J 24: 241-245, 2009.

5. Li H, Wang Q, Gao F, et al: Reduced expression of PDCD5 is associated with high-grade astrocytic gliomas. Oncol Rep 20: 573-579, 2008.

6. Ruan GR, Chen SS, Ma X, et al: Abnormal expression of PDCD5 in the bone marrow cells of adult acute myeloid leukemia. Zhongguo Shi Yan Xue Ye Xue Za Zhi 15: 462-465, 2007.

7. Yang YH, Zhao M, Li WM, Lu YY, Chen YY, Kang B and $\mathrm{Lu}$ YY: Expression of programmed cell death 5 gene involves in regulation of apoptosis in gastric tumor cells. Apoptosis 11: 993-1001, 2006.

8. Ruan GR, Zhao HS, Chang Y, et al: Adenovirus-mediated PDCD5 gene transfer sensitizes K562 cells to apoptosis induced by idarubicin in vitro and in vivo. Apoptosis 13: 641-648, 2008.

9. Yin A, Jiang Y, Zhang X, Zhao J and Luo H: Transfection of PDCD5 sensitizes colorectal cancer cells to cisplatin-induced apoptosis in vitro and in vivo. Eur J Pharmacol 649: 120-126, 2010.

10. Liu J, Li X, Gui R, Jiang T and Wang E: Effect of human recombinant PDCD5 protein on cell apoptosis of multiple myeloma KM3 cells induced by dexamethasone and its mechanism.Zhong Nan Da Xue Xue Bao Yi Xue Ban 35: 725-731, 2010.

11. Shi L, Song Q, Zhang Y, et al: Potent antitumor activities of recombinant human PDCD5 protein in combination with chemotherapy drugs in K562 cells. Biochem Biophys Res Commun 396: 224-230, 2010.

12. Chen C, Zhou H, Xu L, et al: Recombinant human PDCD5 sensitizes chondrosarcomas to cisplatin chemotherapy in vitro and in vivo. Apoptosis 15: 805-813, 2010.

13. Spinola M, Meyer P, Kammerer S, et al: Association of the PDCD5 locus with lung cancer risk and prognosis in smokers. J Clin Oncol 24: 1672-1678, 2006.

14. Ma X, Ruan G, Wang Y, et al: Two single-nucleotide polymorphisms with linkage disequilibrium in the human programmed cell death 5 gene 5 ' regulatory region affect promoter activity and the susceptibility of chronic myelogenous leukemia in Chinese population. Clin Cancer Res 11: 8592-8599, 2005.

15. Nanba K, Toyooka S, Soh J, et al: The allelic distribution of a single nucleotide polymorphism in the PDCD5 gene locus of Japanese non-small cell lung cancer patients. Mol Med Rep 1: 667-671, 2008.

16. Chen Y, Sun R, Han W, Zhang Y, Song Q, Di C and Ma DL: Nuclear translocation of PDCD5 (TFAR19): an early signal for apoptosis? FEBS Lett 509: 191-196, 2001.

17. Chen LN, Wang Y, Ma DL and Chen YY: Short interfering RNA against the PDCD5 attenuates cell apoptosis and caspase-3 activity induced by Bax overexpression. Apoptosis 11: 101-111, 2006.

18. Yao H, Xu L, Feng Y, Liu D, Chen Y and Wang J: Structurefunction correlation of human programmed cell death 5 protein. Arch Biochem Biophys 486: 141-149, 2009.

19. Salvi M, Xu D, Chen Y, Cabrelle A, Sarno S and Pinna LA: Programmed cell death protein 5 (PDCD5) is phosphorylated by CK2 in vitro and in 293T cells. Biochem Biophys Res Commun 387: 606-610, 2009.

20. Xu L, Chen Y, Song Q, Xu D, Wang Y and Ma D: PDCD5 interacts with Tip60 and functions as a cooperator in acetyltransferase activity and DNA damage-induced apoptosis. Neoplasia 11: 345-354, 2009. 
21. Xu M, Cheng N, Gui L, et al: The 5'-upstream region of human programmed cell death 5 gene contains a highly active TATA-less promoter that is up-regulated by etoposide. Gene 329: 39-49, 2004.

22. Menotti L, Nicoletti G, Gatta V, et al: Inhibition of human tumor growth in mice by an oncolytic herpes simplex virus designed to target solely HER-2-positive cells. Proc Natl Acad Sci USA 106: 9039-9044, 2009

23. Santhosh CV, Tamhane MC, Kamat RH, Patel VV and Mukhopadhyaya R: A lentiviral vector with novel multiple cloning sites: stable transgene expression in vitro and in vivo. Biochem Biophys Res Commun 371: 546-550, 2008.

24. Ravet E, Lulka H, Gross F, Casteilla L, Buscail L and Cordelier P. Using lentiviral vectors for efficient pancreatic cancer gene therapy. Cancer Gene Ther 17: 315-324, 2010.

25. Trobridge GD, Beard BC, Gooch C, et al: Efficient transduction of pigtailed macaque hematopoietic repopulating cells with HIV-based lentiviral vectors. Blood 111: 5537-5543, 2008.
26. Hunter MJ, Zhao H, Tuschong LM, Bauer TR Jr, Burkholder TH, Persons DA and Hickstein DD: Gene therapy for canine leukocyte adhesion deficiency with lentiviral vectors using the murine stem cell virus and human phosphoglycerate kinase promoters. Hum Gene Ther 22: 689-696, 2011.

27. Benabdellah K, Cobo M, Muñoz P, Toscano MG and Martin F: Development of an all-in-one lentiviral vector system based on the original TetR for the easy generation of Tet-ON cell lines. PLoS One 6: e23734, 2011.

28. Xie M, Niu JH, Chang Y, et al: A novel triple-regulated oncolytic adenovirus carrying PDCD5 gene exerts potent antitumor efficacy on common human leukemic cell lines. Apoptosis 14: 1086-1094, 2009.

29. Yu ST, Yang YB, Liang GP, et al: An optimized telomerasespecific lentivirus for optical imaging of tumors. Cancer Res 70: 2585-2594, 2010. 